\title{
Leader
}

\section{Serum markers in myocardial infarction}

\author{
Nikhil R Patel, Graham Jackson
}

Rapid and accurate diagnosis of chest pain is a routine requirement in emergency departments. Thrombolysis or immediate coronary angioplasty, or both, for evolving myocardial infarction has been shown to reduce morbidity and mortality but the benefit is time dependent. $^{1}$

Between $70 \%$ and $80 \%$ of patients with an acute myocardial infarct have ischaemic chest pain at presentation, but only $25 \%$ of those admitted with chest pain will have an infarct confirmed. Unstable angina will be the diagnosis in a further $30 \%$, which means that a significant number of patients are unnecessarily admitted. Conversely $5 \%$ will be sent home inappropriately because of the diagnostic limitations of the ECG and creatine kinase-MB (CK-MB) measurements. Clearly there will be no need for laboratory confirmation of the diagnosis if the ECG identifies acute myocardial infarction but the initial ECG is only abnormal in $50 \%$ of cases. Waiting for change in serial ECGs introduces delay. The need for an early accurate laboratory marker has become an essential biochemical quest in the era of near patient testing.

CK-MB has been our gold standard serum marker for myocardial infarction but it lacks specificity for cardiac muscle and an MB above the normal range may not be seen for six to eight hours after the onset of an acute myocardial infarct. ${ }^{2}$ CK-MB isoforms using a 25 minute assay have reduced the time to abnormality to two hours. ${ }^{3}$ The sensitivity is twice that of conventional CK-MB and when taking serial measurements every 30 to 60 minutes until at least six hours after symptoms, the sensitivity to detect acute myocardial infarction at six hours increases to $95.7 \%$ compared with $48 \%$ for conventional CK-MB. The commonest cause of false positive results is unstable angina causing minor myocardial injury. These increases may be missed by conventional CK-MB, which in turn has failed to identify those with unstable angina who are at increased risk of subsequent cardiac events in the six months following initial presentation. Finally a negative subform assay is not reliable unless the blood sample is taken at least six hours after the onset of chest pain. While this may lead to patients spending longer in the emergency department it should be remembered that most arrive after a considerable delay so the wait for a negative result is likely to be less than four hours.

Troponin $\mathrm{T}$ and I are part of a complex of proteins that regulate the calcium mediated interaction of actin and myosin in cardiac as well as skeletal muscle. ${ }^{4}$ Using assays based on monoclonal antibodies against the cardiac isoforms (C-TnT, c-TnI) there is no significant skeletal cross reactivity. They are more specific than CK-MB for cardiac injury and, because they are not found in the blood of normal healthy people, detection of minor cell damage in unstable patients is more likely to occur. Importantly, though this reduces the specificity for the detection of acute myocardial infarction, trials have shown that raised troponin values in acute coronary syndromes (for example, unstable angina, non-Q-wave infarction) predict subsequent death and myocardial infarction. ${ }^{56}$ Troponin I is the most specific for cardiac muscle and in contrast to troponin $\mathrm{T}$ it is not influenced by renal disease (false positives). Troponins become raised three to 12 hours after the onset of symptoms and remain raised for five to 10 days, in contrast to 48 to 72 hours for CK-MB.

A recent study of over 700 patients presenting to the emergency room with chest pain of less than 12 hours' duration examined the value of rapid bedside tests for troponins $T$ and I, measured on arrival and four hours later. ${ }^{7}$ One positive test was recorded in $16 \%$ of cases on admission and in $22 \%$ at four hours. No cases of acute myocardial infarction were missed (compared with the accepted rate of $5 \%)$. In addition, troponins $\mathrm{T}$ and I were superior to CK-MB and the ECG at independently predicting subsequent cardiac events.

This study improves on the diagnostic accuracy of the CK-MB isoforms, allowing early discharge in the presence of two carefully timed negative troponin tests, with important resource implications. One positive test selects high risk and the need for hospital admission, and possibly-though not proven-benefit from intervention (trials are needed). While false positive results are unusual, myocarditis, pulmonary embolism, and cardiac trauma may raise troponins by causing direct myocardial injury. The possibility that raised troponins after non-cardiac surgery predict subsequent ischaemic events in high risk individuals warrants further study. ${ }^{8}$ 
When the patient presents in the emergency room with chest pain, laboratory tests complement the initial clinical assessment and ECG. Rapid bedside measurements of troponin $\mathrm{T}$ or I (12 to 15 minutes) will need to be accurately read, but provide clear guidance on who is at risk. There remains, however, a delay that needs to be overcome if we are to maximise the benefits of modern coronary care.

Myoglobin is increased in 30\% of cases within two hours of acute myocardial infarction and at this time it is superior to CK-MB in diagnostic sensitivity. Later the sensitivity is reduced owing to rapid renal clearance. A 10 minute emergency room test is available, but there is concern over its low specificity. ${ }^{9}$

Troponins have the advantage of being the most specific markers for myocardial injury, though not the fastest. They also provide evidence of a myocardial infarct which may have developed up to 10 days earlier, in contrast to the other markers which will be negative at this point. Use of either troponin marker is suggested by the American Heart Association. At the moment troponin $\mathrm{T}$ is the most validated and the evidence of its ability to rule in or rule out myocardial damage cannot be disputed. ${ }^{10}$ In the unstable patient, absence of a rise in troponin does not identify no risk, but points to the need for a non-urgent assess- ment, for example by exercise testing or nuclear studies.

It is "time for $\mathrm{T}$ " but only combined with clinical acumen and the ECG, and we still have to accurately close the $0-3$ hour time window.

1 Ryan TJ, Anderson JL, Antman EM, et al. ACC/AHA guidelines for the management of patients with acute myocardial infarction. $\mathcal{F}$ Am Coll Cardiol 1996;28:1328-428.

2 Hamm CW. New serum markers for acute myocardial Hamm CW. New serum markers for a
infarction. N Engl f Med 1994;331:607-8.

3 Puleo PR, Meyer D, Wathen C, et al. Use of a rapid assay of subforms of creatinine kinase MB to diagnose or rule out acute myocardial infarction. $N$ Engl f Med 1994;331:5616.

4 Van de Werf F. Cardiac troponins in acute coronary syndrome. N Engl f Med 1996;335:1388-9.

5 Ohman EM, Armstrong PW, Christenson RH, et al. Cardiac Ohman EM, Armstrong PW, Christenson RH, et al. Cardiac
troponin T levels for risk stratification in acute myocardial ischaemia. N Engl f Med 1996;335:1333-41.

6 Antman EM, Tanasijevic MJ, Thompson B, et al. Cardiacspecific troponin I levels to predict risk of mortality in patients with acute coronary syndrome. $N$ Engl f Med 1996;335:1342-9.

7 Hamm CW, Goldman BU, Heeschen C, et al. Emergency room triage of patients with acute chest pain by means of rapid testing for cardiac troponin T or troponin I. $N$ Engl F Med 1997;337:1648-53.

8 Lopez-Jimenez F, Goldman L, Sacks DB, et al. Prognostic value of cardiac troponin $\mathrm{T}$ after non-cardiac surgery: 6-month follow-up data. $7 \mathrm{Am}$ Coll Cardiol 1997;29:12415.

9 Solymoss BC, Bourassa MG, Wesolowska E, et al. The role of cardiac troponin $\mathrm{T}$ and other new biochemical markers
in evaluation and risk stratification of patients with acute chest pain syndrome. Clin Cardiol 1997;20:934-42.

10 Stubbs P, Collinson P, Moseley D, et al. The prognostic significance of admission troponin $\mathrm{T}$ concentrations in myocardial infarction. Circulation 1996;94:1291-7. 